\title{
Does SARS-CoV-2 affect cochlear functions in children?
}

Cigdem F. Koca, MD, Turgut Celik, MD, Agit Simsek, PhD, Sukru Aydin, MD, Mehmet Kelles, MD, Seyma Yasar, PhD, Omer Erdur, $M D$.

\begin{abstract}

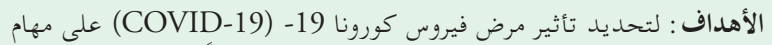

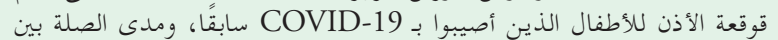
خطورة المرض ومشاركة القوقعة في الانبعاثات الصوتية (OAEs)

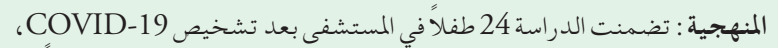

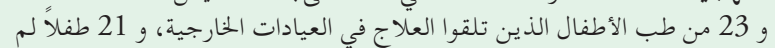

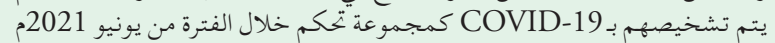

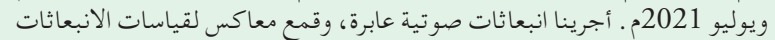

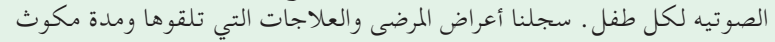
الأطفال في المستشفى في المجموعة. سجلنا لمراض المرض

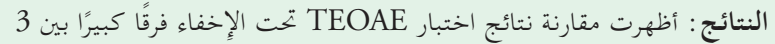

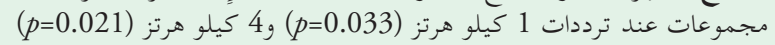

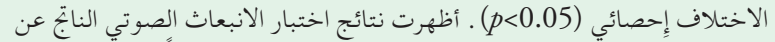

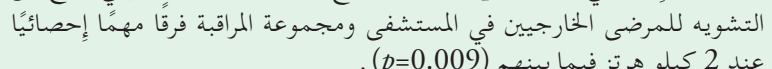
الحخلاصة : تشير نتائجنا إلى أن فيروس كورونا - 2 المتلازمة التنفسية الحادة

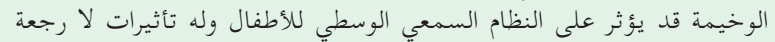

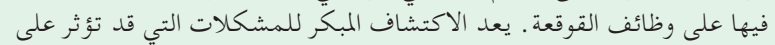

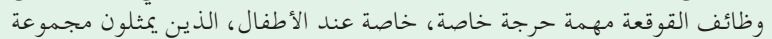

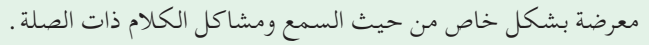

Objectives: To determine the influence of coronavirus disease-19 (COVID-19) on cochlear tasks of children who had COVID-19 previously, and the relevance among disease seriousness and cochlear involvement by otoacoustic emissions (OAEs).

Methods: The study included 24 hospitalized children after COVID-19 diagnosis, 23 pediatrics that received outpatient treatment, and 21 children who were without COVID-19 diagnosis as the control group between June 2021 and July 2021. Transient evoked otoacoustic emission (TEOAE), distortion product otoacoustic emission, and contrlateral suppression of otoacoustic emission measurements were carried out for each child. Symptoms of patients, the treatments they received, and the duration of hospitalization of the children in the hospitalized group were recorded.

Results: The comparison of TEOAE test results under masking showed a considerable difference between
3 groups at $1 \mathrm{kHz}(p=0.033)$ and $4 \mathrm{kHz}(p=0.021)$ frequencies $(p<0.05)$. Distortion product otoacoustic emission test results of hospitalized outpatient and control group showed a statistically significant difference at $2 \mathrm{kHz}$ among themselves $(p=0.009)$.

Conclusion: Our results suggest that severe acute respiratory syndrome coronavirus- 2 may influence the medial olivocochlear system of children and have irreversible effects on the cochlear functions. Early detection of problems that may affect cochlear functions is a special critical task, especially in children, who are a particularly vulnerable group in terms of hearing and related speech problems.

Keywords: COVID-19, cochlear, SARS-CoV-2, pediatric

Saudi Med J 2022; Vol. 43 (3): 259-265

doi: 10.15537/smj.2022.43.3.20210782

From the Department of Otolaryngology Head and Neck Surgery (Koca, Kelles), Faculty of Medicine, Turgut Özal University, from the Department of Otolaryngology Head and Neck Surgery (Celik, Aydin), Malatya Training and Research Hospital, from the Department of Audiology (Simsek), Faculty of Health Sciences; from the Department of Biostatistics and Medical Informatics (Yasar), Faculty of Medicine, Inonu University, Malatya, and from the Department of Otorhinolaryngology (Erdur), Faculty of Medicine, Selcuk University, Konya, Turkey.

Received 27th September 2021. Accepted 26th December 2021.

Address correspondence and reprint request to: Dr. Cigdem F. Koca, Department of Otolaryngology Head and Neck Surgery, Faculty of Medicine, Turgut Ozal University, Malatya, Turkey. E-mail: cifirat@hotmail.com

ORCID ID: https://orcid.org/0000-0001-8990-0651

C oronavirus disease-2019 (COVID-19) is a recent and quite contagious illness and it presents itself with various kinds of complaints, including respiratory symptoms, neurological, peripheral nervous complaints, and skeletal muscle findings. ${ }^{1}$ Although the symptoms of COVID-19 is mainly in respiratory nature, increasing neurological reports up to $30 \%$ of cases showed that 
severe acute respiratory syndrome coronavirus-2 (SARS-CoV-2) has a neurotropic character. ${ }^{2}$ In postmortem studies, genome sections were determined in brain tissues of infected patients, with proof of several pathological alterations in cortical neurons, hypothalamus, as well as cerebrospinal fluids. ${ }^{3}$ Smell and taste dysfunctions, impaired consciousness, headache, and dizziness are some neurological symptoms that have been reported with COVID-19 patients during the pandemic. ${ }^{4-11}$ Additionally, the disease can also devastate the hearing organs. Chirakkal et $\mathrm{al}^{1}$ presented a case that was suffering from hearing loss and tinnitus during COVID-19, and they underlined the significance of audiological evaluation. They could not obtain lowfrequency thresholds in pure-tone audiometry and responses from otoacoustic measurements. These results showed the detrimental effects of COVID-19 in the cochlear outer hair cells. Human immunodeficiency virus, hepatitis, measles, herpes simplex, enteroviruses, mumps, rubella, and lassa could influence cranial nerves that results in taste and smell dysfunctions, facial paralysis, and sudden sensorineural hearing loss (SSNHL). Neuritis and cochleitis may occur due to the cochlear neural viral invasion. Viral infestation of cochlea and perilymphatic structures by stress as an answer to the cross interaction between the inner ear antigenic structures and the viruses creates the primary pathogenesis of virus originated SSNHL. Autopsy is the exact route to prove the viral neural damage. Autopsy procedure of the SARS-CoV-2 infected cases showed more hyperemic as well as edematous featured brain tissue and concurrent neuronal injury. ${ }^{12-14}$ Outer hair cells creates otoacoustic emissions (OAE); and OAEs can be measured from external ear canal. Administration of a sound contralateral or ipsilateral to a healthy ear from that OAEs being measured, decreases or supresses the OAE amplitudes. This suppression of $\mathrm{OAE}$ due to sound is a physiological mechanism carried out through efferent auditory mechanism. The medial olivocochlear (MOC) fibers anatomically arise from the medial nuclear part of superior olivary complexity. Contralateral acoustic stimulation activates the bundle and this activation concludes with termination of cochlear functions commonly through outer hair cells. The absence of contralateral suppression (CS) is an abnormal result that indicates the deficiency of the efferent auditory mechanism. ${ }^{15-17}$

Disclosure. Authors have no conflict of interests, and the work was not supported or funded by any drug company.
Coronavirus disease-2019 morbidity and mortality have been lower in pediatric patients compared to the elderly. However, there are still not enough studies in pediatric patients. Studies are required in children with COVID-19 to identify possible damages and discuss early intervention if necessary. In the present study, our aim was to evaluate whether COVID-19 has any influence on cochlear functions of children who had previous COVID-19 history, and the relationship among disease intensity and cochlear involvement by OAEs.

Methods. This prospective clinical research was carried out in the Otolaryngology Department, Malatya Training and Research Hospital, Malatya, Turkey, and was carried out on children who were diagnosed with COVID-19 and recovered at least 3 months before the study date. The study included 24 hospitalized children with COVID-19 diagnosis between June 2021 and July 2021. A total of 23 children that received outpatient treatment and 21 children who were admitted to the ENT clinic for different reasons without having COVID-19 diagnosis with similar age and gender were distributed as the control group. A complete otolaryngological examination was carried out for all patients. Acoustic reflexes and tympanogram were measured from both right and left ears with $226 \mathrm{~Hz}$ probe tone frequency for all children. Inclusion criteria included patients who had ear diseases (middle or outer), children who could not succeed neonatal hearing screening program, those who required intensive care support in postnatal period, those who had family history of hereditary character hearing loss, children whose mother experienced toxoplasmosis, rubella cytomegalovirus, herpes simplex, and HIV infections in the course of pregnancy, those with head and neck abnormalities, including auricular and external auditory canal deformities, and whose birth weight were under 1500 grams, children who have not completed 37 weeks (premature born), and those diagnosed with severe hyperbilirubinemia.

Distortion product otoacoustic emission (DPOAE), Transient evoked otoacoustic emission (TEOAE), and contrlateral suppression (CLS) of OAE tests were carried out for all participants. Symptoms of all patients, the treatments they received, and the duration of hospitalization of the children in the hospitalized group were recorded. Exclusion criteria included healthy children with normal tympanogram (type A) and who succeed neonatal hearing screening program.

OtoAccessTM database software program (Interacoustics, Middelfart, Denmark) and EP15 Eclipse Module test device were used to assess TEOAE binaurally. Noise, signal, signal-to-noise ratio (S/N-R) 
parameters were measured in the TEOAE test. Transient evoked otoacoustic emission measurements were carried out at the following frequencies: 1000-1500 and 2000-3000 and $4000 \mathrm{~Hz}$. A linear click TEOAE stimulus ( $80 \pm 3 \mathrm{~dB}$ SPL intensity) was applied for one ear. Stimulus stability value was greater than $80 \%$. The existence of TEOAE test procedure is normal. Reconstructed reproducibility was admitted in cases where it was better than $70 \%$.

The database program used in the TEOAE test module and the same OAE device were also used for the DPOAE test. The same test parameters that we used in the TEOAE test were examined. In DPOAE measurement, 2 synchronous pure-tone signals were applied to the ear at 2 different frequencies ( $\mathrm{f} 1$ and $\mathrm{f} 2$, $\mathrm{f} 2>\mathrm{f} 1$ ). Measurements were detected with a frequency ratio $\mathrm{f} 2 / \mathrm{f} 1$ set at 1.22 .

Transient evoked otoacoustic emission test variables were utilized for the assessment of CLS with TEOAE testing procedure. Assessment was carried out for both right and left ears. A linear click TEOAE stimulus at $80 \pm 3 \mathrm{~dB}$ SPL intensity was applied from one ear, and a $60 \mathrm{~dB}$ SPL white noise was given to the opposite ear in a linear stimulus format in order to supress the efferent auditory system and then the TEOAE measurement process was repeated. White noise stimulus to be delivered contralaterally via placed earphones. We compared the TEOAE results with the responses occured due to the contralateral suppression by assessing both of them binaurally. Signal, noise, and S/N-R were used as research components. Signal and S/N-R suppression amplitudes were counted up by subtracting the TEOAE outcomes obtained in the existence of noise from the TEOAE responses measured under silent. Positive supression amplitudes obtained according to the calculation mentioned above accepted as 'suppression exists' and negative supression amplitudes were categorized as 'no suppression'. The signal suppression frequency was measured for all children.

Informed written and verbal consent were obtained from parents of all children. This prospective research was designed in a blind attitude, that the authorized audiologist who carried out the OAE tests was uninformed on whether the children were member of the normal group or had COVID-19 previously. We carried out this clinical trial after the approval of the Turkish Ministry of Health and the Malatya Clinical Research Ethics, Malatya, Turkey (ethical no.: 2021/36). The clinical research was carried out according to the Helsinki Declaration.

Statistical analysis. Results were summed up as mean \pm standard deviation (SD), median (min-max), and count (percentage). Relevance to the normative dispersion was carried out by the Shapiro-Wilk test. One-way ANOVA was used for the variables that provided the assumption of normal distribution in the statistical analysis, and the Kruskall-Wallis test was used for the data that did not provide the assumption of normal distribution. Bonferroni correction was used in multiple comparisons among clusters. Pearson Chi-square test was carried out to analyse the connection among qualitative components and groups. Analysis was carried out by the Statistical Package for the Social Sciences, version 26 (IBM Corp., Armonk, NY, USA). A $p$-value of $<0.05$ was considered significant.

Results. The average age of hospitalized pediatrics was calculated as 33.6 months (35 months for outpatients and 35.4 months for control group). Gender distribution were similar between groups. The mean stay of hospitalized children was 3.5 days. The time between the test and COVID-19 was 128.4 days in the hospitalized group and 119.4 in the outpatients group. Demographic data was similar for both groups $(p>0.05)$. When the symptoms of the patients during the COVID-19 process were examined, fever, cough, dyspnea, diarrhea, nausea, vomiting, poor oral intake, nasal congestion, headache, rash, and sore throat were observed. Fever was the most common symptom in both groups. Symptoms such as fever, cough, dyspnea, and poor oral intake were more common in the inpatient group, indicating that the disease was more severe. When the hospitalized group and outpatients group were compared in terms of symptoms related to COVID-19, dyspnea was solely differed between the groups $(p=0.048)$.

Transient evoked otoacoustic emission test amplitudes obtained in silence were compared for the 3 groups (hospitalized patients, outpatients, and control group), a significant difference was not found in any frequency $(p>0.05$; Table 1$)$. When the results of the TEOAE test under masking were analyzed, amplitudes of $1 \mathrm{kHz}(p=0.033)$ and $4 \mathrm{kHz}(p=0.021)$ frequencies were differed among the 3 groups $(p<0.05$; Table 2). Comparison of TEOAE test results for $1 \mathrm{kHz}$ amplitudes between control and hospitalized $(p=0.018)$ and inpatient-outpatient $(p=0.031)$ paired groups showed difference. The relationship between the control - outpatient groups was insignificant $(p=0.857)$. At $4 \mathrm{kHz}$ the difference between the control - hospitalized patient groups $(p=0.006)$ showed statistical significance but the difference between the hospitalized patient - outpatient $(p=0.063)$ and control - outpatient ( $p=0.393)$ groups were both statistically insignificant. According to the statistically significant results at these 
frequencies, amplitudes were found to be higher in children with COVID-19 maybe due to the efferent system dysfunction.

The comparison of the contralateral suppression amplitudes at any frequency for the 3 groups were insignificant $(p>0.05$; Table $3 \&$ Figure 1$)$. The comparison of the contralateral suppression OAE test results of hospitalized and outpatient children and control group was numerically insignificant $(p>0.05$; Table 4).

Distortion product otoacoustic emission test results of hospitalized, outpatient and control group showed a statistically significant difference at $2 \mathrm{kHz}$ among themselves ( $p=0.009$; Table 5). In paired groups according to the DPOAE test results at $2 \mathrm{kHz}$, the difference between the control - hospitalized patients $(p=0.020)$ and control - outpatient $(p=0.003)$ groups were statistically significant, but the comparison between the hospitalized patient - outpatient groups was insignificant $(p=0.491)$.
Discussion. Based on the outcomes of this study, our research indicated a statistically significant difference between hospitalized and outpatient children and control group in TEOAE (obtained under masking) test at $1 \mathrm{kHz}(p=0.033)$ and $4 \mathrm{kHz}$ $(p=0.021)$ frequencies. We divided the groups in 2 again to examine the relationship between the difference and disease intensity. Control-hospitalized and hospitalized-outpatient group analysis both showed a statistically significant difference at $1 \mathrm{kHz}$ frequency. This analysis was statistically significant in only controlhospitalized group in comparison at $4 \mathrm{kHz}$ frequency $(p=0.006)$. Accordingly, amplitudes were found to be higher in children with COVID-19 maybe due to the dysfunction of efferent system at these frequencies. It is thought that noise was perceived as an extra stimulus because efferent system could not carry out appropriate suppression. Additionally, the comparison of DPOAE test results at $2 \mathrm{kHz}$ frequency between the 3 groups showed a considerable difference $(p=0.009)$. Similar to

Table 1 - Transient evoked otoacoustic emission results measured in silence.

\begin{tabular}{|c|c|c|c|c|c|c|c|}
\hline \multirow[t]{3}{*}{ Frequencies } & \multicolumn{6}{|c|}{ Groups } & \multirow[t]{3}{*}{$P$-value } \\
\hline & \multicolumn{2}{|c|}{ Hospitalized patients } & \multicolumn{2}{|c|}{ Outpatients } & \multicolumn{2}{|c|}{ Controls } & \\
\hline & Mean \pm SD & Median (min-max) & Mean \pm SD & Median (min-max) & Mean \pm SD & Median (min-max) & \\
\hline $1 \mathrm{kHz}$ & $6.6 \pm 2.99$ & $7(-3-14)$ & $5.89 \pm 3.55$ & $5(-2-16)$ & $6.5 \pm 4.22$ & $6(-3-14)$ & $0.409^{*}$ \\
\hline $1.5 \mathrm{kHz}$ & $8.23 \pm 3.95$ & $8(-3-20)$ & $7.13 \pm 3.19$ & $7.5(1-15)$ & $9.06 \pm 4.85$ & $9(-2-22)$ & $0.087^{* *}$ \\
\hline $2 \mathrm{kHz}$ & $7.42 \pm 3.48$ & $7(-2-14)$ & $7.72 \pm 3.8$ & $7(3-24)$ & $6.88 \pm 4.65$ & $8(-4-15)$ & $0.949^{*}$ \\
\hline $3 \mathrm{kHz}$ & $6.71 \pm 4.04$ & $7(-2-18)$ & $6.91 \pm 4.31$ & $7(-3-20)$ & $7.24 \pm 3.81$ & $6(0-17)$ & $0.897^{* *}$ \\
\hline $4 \mathrm{kHz}$ & $6.92 \pm 3.69$ & $7(-3-16)$ & $6.3 \pm 3.93$ & $6(-2-14)$ & $5.46 \pm 3.94$ & $6(-4-12)$ & $0.398^{*}$ \\
\hline
\end{tabular}

kHz: kilohertz, SD: standard deviation, Min: minimum, Max: maximum, "Kruskall-Wallis, * one-way ANOVA, a statistically significant difference $(p<0.05)$

Table 2 - Transient evoked otoacoustic emission results obtained through masking.

\begin{tabular}{|c|c|c|c|c|c|c|c|}
\hline \multirow[t]{3}{*}{ Frequencies } & \multicolumn{6}{|c|}{ Groups } & \multirow[t]{3}{*}{$P$-value } \\
\hline & \multicolumn{2}{|c|}{ Hospitalized patients } & \multicolumn{2}{|c|}{ Outpatients } & \multicolumn{2}{|c|}{ Controls } & \\
\hline & Mean \pm SD & Median (min-max) & Mean \pm SD & Median (min-max) & Mean \pm SD & Median (min-max) & \\
\hline $1 \mathrm{kHz}$ & $4.67 \pm 2.61$ & $4.5(-5-10)$ & $2.8 \pm 5.05$ & $3(-9-20)$ & $2.42 \pm 4.55$ & $3.5(-9-10)$ & $0.033^{*}$ \\
\hline $1.5 \mathrm{kHz}$ & $5.38 \pm 3.46$ & $5(-1-16)$ & $4.67 \pm 3.55$ & $4(-5-14)$ & $5.28 \pm 4.38$ & $4(-4-18)$ & $0.432^{*}$ \\
\hline $2 \mathrm{kHz}$ & $5.46 \pm 2.82$ & $5.5(-3-11)$ & $5.3 \pm 3.2$ & $5(-6-12)$ & $4.08 \pm 4.38$ & $4(-5-12)$ & $0.209^{*}$ \\
\hline $3 \mathrm{kHz}$ & $4.94 \pm 3.13$ & $5(-4-13)$ & $4.61 \pm 4.26$ & $5(-9-18)$ & $4.32 \pm 3.29$ & $4(-6-12)$ & $0.384^{*}$ \\
\hline $4 \mathrm{kHz}$ & $4.85 \pm 3.16$ & $5(-5-11)$ & $3.33 \pm 4.49$ & $4(-6-13)$ & $2.4 \pm 4.16$ & $4(-5-10)$ & $0.021^{*}$ \\
\hline
\end{tabular}

kHz: kilohertz, SD: standard deviation, Min: minimum, Max: maximum, ${ }^{\star}$ Kruskall-Wallis, a statistically significant difference $(p<0.05)$ 
SARS-CoV-2 effects on cochlear functions ... Koca et al

Table 3 - Contralateral supression amplitudes.

\begin{tabular}{|c|c|c|c|c|c|c|c|}
\hline \multirow[t]{3}{*}{ Frequencies } & \multicolumn{6}{|c|}{ Groups } & \multirow[t]{3}{*}{$P$-value } \\
\hline & \multicolumn{2}{|c|}{ Hospitalized patients } & \multicolumn{2}{|c|}{ Outpatients } & \multicolumn{2}{|c|}{ Controls } & \\
\hline & Mean \pm SD & Median (min-max) & Mean \pm SD & Median (min-max) & Mean \pm SD & Median (min-max) & \\
\hline $1 \mathrm{kHz}$ & $1.94 \pm 1.12$ & $2(-1-5)$ & $3.09 \pm 3.44$ & $2(-4-13)$ & $4.08 \pm 4.1$ & $2(-2-15)$ & $0.063^{*}$ \\
\hline $1.5 \mathrm{kHz}$ & $2.85 \pm 1.79$ & $2.5(-2-8)$ & $2.46 \pm 1.77$ & $2.5(-1-8)$ & $3.78 \pm 2.96$ & $3(-1-13)$ & $0.097^{*}$ \\
\hline $2 \mathrm{kHz}$ & $1.96 \pm 2.39$ & $1.5(-6-8)$ & $2.41 \pm 2.77$ & $2(-6-13)$ & $2.8 \pm 3.42$ & $1.5(-2-12)$ & $0.594^{*}$ \\
\hline $3 \mathrm{kHz}$ & $1.77 \pm 2.8$ & $2(-7-11)$ & $2.3 \pm 2.64$ & $2(-7-10)$ & $2.92 \pm 2.9$ & $2(0-11)$ & $0.351^{*}$ \\
\hline $4 \mathrm{khz}$ & $2.06 \pm 2.88$ & $2(-5-9)$ & $2.98 \pm 3.07$ & $2.5(-3-10)$ & $3.06 \pm 3.52$ & $2(-10-12)$ & $0.284^{*}$ \\
\hline
\end{tabular}

kHz: kilohertz, SD: standard deviation, Min: minimum, Max: maximum, "Kruskall-Wallis, a statistically significant difference $(p<0.05)$

10.00

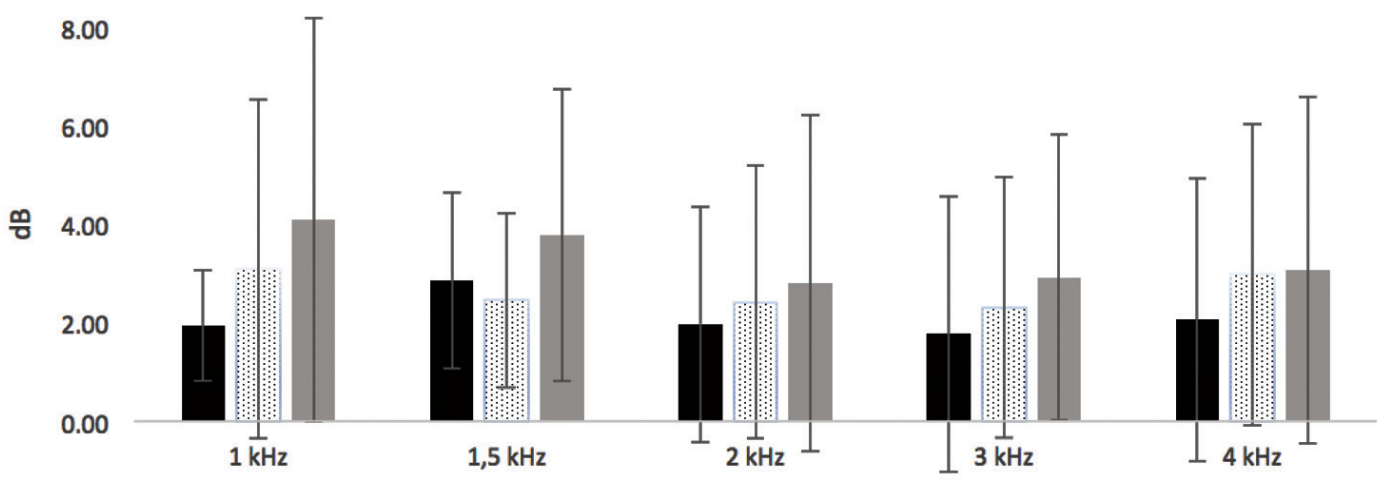

$-2.00$

- Hospitalized Patient Outpatient Control

Figure 1 - Contralateral supression amplitudes. dB: decibel, kHz: kilohertz

Table 4 - Contralateral supression amplitudes.

\begin{tabular}{|c|c|c|c|c|c|}
\hline \multirow[t]{2}{*}{ Frequencies } & \multirow[t]{2}{*}{ Suppression } & \multicolumn{3}{|c|}{ Groups } & \multirow[t]{2}{*}{$P$-value } \\
\hline & & Hospitalized patients & $\begin{array}{l}\text { Outpatients } \\
\text { n (\%) }\end{array}$ & Controls & \\
\hline \multirow{2}{*}{$1 \mathrm{kHz}$} & No & $3(6.2)$ & $7(15.2)$ & $3(6.0)$ & \multirow{2}{*}{$0.206^{*}$} \\
\hline & Yes & $45(93.7)$ & $39(84.8)$ & $47(94.0)$ & \\
\hline \multirow{2}{*}{$1.5 \mathrm{kHz}$} & No & $1(2.1)$ & $4(8.7)$ & $1(2.0)$ & \multirow{2}{*}{$0.176^{*}$} \\
\hline & Yes & 47 (97.9) & $42(91.3)$ & $49(98.0)$ & \\
\hline \multirow{2}{*}{$2 \mathrm{kHz}$} & No & $9(18.7)$ & $6(13.0)$ & $9(18.0)$ & \multirow{2}{*}{$0.723^{*}$} \\
\hline & Yes & $39(81.2)$ & $40(87.0)$ & $41(82.0)$ & \\
\hline \multirow{2}{*}{$3 \mathrm{kHz}$} & No & $11(22.9)$ & $7(15.2)$ & $4(8.0)$ & \multirow{2}{*}{$0.122^{*}$} \\
\hline & Yes & $37(77.1)$ & $39(84.8)$ & $46(92.0)$ & \\
\hline \multirow{2}{*}{$4 \mathrm{kHz}$} & No & $13(27.1)$ & $9(19.6)$ & $5(10.0)$ & \multirow{2}{*}{$0.094^{*}$} \\
\hline & Yes & $35(72.9)$ & $37(80.4)$ & $45(90.0)$ & \\
\hline
\end{tabular}


Table 5 - Distortion product otoacoustic emission results.

\begin{tabular}{lcccccc}
\hline Frequencies & \multicolumn{2}{c}{ Hospitalized patients } & \multicolumn{2}{c}{ Groups } & \multicolumn{2}{c}{$P$-value } \\
& Mean \pm SD & Median (min-max) & Mean \pm SD & Median (min-max) & Mean \pm SD & Median (min-max) \\
\hline $1 \mathrm{kHz}$ & $8.54 \pm 3.42$ & $8.15(1.4-23.6)$ & $9.15 \pm 4.62$ & $8.25(1.4-31.1)$ & $9.48 \pm 4.42$ & $10(-1-18.4)$ \\
$2 \mathrm{kHz}$ & $12.41 \pm 5.92$ & $10.9(1.3-27.5)$ & $11.54 \pm 5.24$ & $10.3(1.1-26.4)$ & $14.61 \pm 5.09$ & $14.65(7.7-30.4)$ \\
$4 \mathrm{kHz}$ & $14.68 \pm 7.09$ & $12.9(2.2-34.6)$ & $14.04 \pm 6.2$ & $12.75(5.1-28)$ & $16.13 \pm 6.5$ & $0.080^{*}$ \\
$6 \mathrm{kHz}$ & $12.68 \pm 5.45$ & $11.75(3.1-25.1)$ & $11.45 \pm 5.45$ & $10.6(1.3-24.1)$ & $10.98 \pm 4$ & $109^{*}$ \\
& & & & &
\end{tabular}

kHz: kilohertz, SD: standard deviation, Min: minimum, Max: maximum, "Kruskall-Wallis, a statistically significant difference $(p<0.05)$

the TEOAE test, we investigated whether this difference was related to disease severity of DPOAE test results. Distortion product otoacoustic emission measurements were differed between only the control and hospitalized groups ( $p=0.006)$.

While this study test results were not differed between groups, we believed that SARS-CoV-2 may have affected the cochlear functions in pediatric patients. The severity of the disease increased, considering that the group of hospitalized patients had a more serious illness, the possibility of COVID-19 injury on cochlear functions increased as well.

The efferents of MOC emerge in the medial portion of the superior olivary complexity, travel via vestibular nerve and end on the outer hair cells. Activation of MOC efferent fibers provides an inactivation on the outer hair cells activity and a decrease in the OAE amplitudes. Otoacoustic emissions are created through cochlear outer hair cells, in a spontaneous way or as a reaction to acoustic stimulus, and it can be measured from the external ear canal easily. Contralateral acoustic stimulation and OAEs provide the evaluation of the efferent cochlear functions. This phenomenon is defined as CLS of OAEs and it eases the determination of the MOC functions efferent system. ${ }^{15-17}$

Medial olivocochlear efferent system has some critical tasks in hearing processes. It supports auditory perception in noisy environment via anti-masking and this complex mechanism maintains improved tone determination, intensity, and speech discrimination. It arranges selective attention by regulating outer hair cells' functions in the ear to which the notice is targeted. Additionally, MOC protects the inner ear from acoustic damage. ${ }^{18}$ Medial olivocochlear reflex provides the auditory mechanisms to accommodate noises by decreasing its reflection to a fixed noise existence. This process is essential to orient the dynamic human hearing to the environment. The contralateral MOC system efferents affect the functions of the outer hair cells directly. In normal healthy individuals, contralateral noise enhances the capacity to understand speech in noise. The impaired MOC reflexes may cause impairment in language acquisition via disrupting the distinguishing of speech sounds from noises in the vital period of language acquisition, that could not be detectable in hearing thresholds. ${ }^{19}$ Auditory system injury due to viral diseases is mainly intracochlear. On the other hand, some viral agents may affect both cochlea and auditory brainstem. Viruses can use some mechanisms to create the damage on the peripheral auditory system including direct injury on the stria vascularis, spiral ganglion or organ of Corti, immunemediated injury arranged by the immune mechanism of the patient as a response to the virally produced proteins, and susceptibility to secondary bacterial infections of the ear. Otolaryngologists should be reminded that COVID-19 may emerge with wide variety of symptoms and lack of major symptoms hide unclear effect of the disease on vulnerable organs such as the cochlea. ${ }^{1}$

Hearing impairments may be based on vascular injury because hearing organs are vulnerable to ischemic conditions in consequence of terminal vasculature structure and extra energy necessitate. ${ }^{2}$ The number of studies that were revealing the effect of COVID-19 on cochlear activity were quite limited in the literature. Mustafa et $\mathrm{al}^{20}$ introduced the destructive effects of COVID-19 on outer hair cells. He demonstrated this injury via the decreased TEOAE amplitudes recorded from patients with COVID-19 compared with healthy participants. Kilic et $\mathrm{al}^{14}$ presented a series of 5 patients whose first and only clinical symptom was SSNHL. Perret et $\mathrm{al}^{21}$ reported an 84-year-old COVID-19 positive patient diagnosed with acute labyrinthitis. They reported that labyrinthitis might be developed due to COVID-19.

In our previous study, we analysed the outcomes of contralateral suppression of OAE measurement of infants who were exposed to the SARS-CoV-2 virus in intrauterine period and compared it with healthy infants. We found that the novel virus SARS-CoV-2, may influence the efferent mechanism of infants, particularly at high frequencies and we have seen an 
insufficiency in MOC system of these infants who were exposed to the SARS-CoV-2 virus in intrauterine period. $^{22}$

Study limitations. Since we did not have antibody tests, we could not test the control group. In order to avoid possible confusion, children who presented with symptoms of COVID-19 or had close contiguity with a SARS-CoV-2 positive patient in the last 6 months were not included in the control group and the small number of our patients limits our study as well. These results need to be supported by a larger patient groups in the future.

In conclusion, according to the outcomes of this study, we have considered that SARS-CoV-2 may influence MOC system of children and have irreversible effects on the cochlear functions. Early detection of problems that may affect cochlear functions is a particularly critical task, especially in children, who are a particularly vulnerable group in terms of hearing and related speech problems. We have searched the literature and found that this is the second study that examines the influences of SARS-CoV-2 on the cochlea, particularly on the pediatric patients.

Acknowledgment. The authors gratefully acknowledge SCRIBENDI (www.scridendi.com) for English language editing.

\section{References}

1. Chirakkal P, Al Hail AN, Zada N, Vijayakumar DS. COVID-19 and tinnitus. Ear Nose Throat J 2021; 100: 160S-162S.

2. Viola P, Ralli M, Pisani D, Malanga D, Sculco D, Messina L, et al. Tinnitus and equilibrium disorders in COVID-19 patients: preliminary results. Eur Arch Otorhinolaryngol 2021; 278: 3725-3730.

3. Paniz-Mondolfi A, Bryce C, Grimes Z, Gordon RE, Reidy J, Lednicky J, et al. Central nervous system involvement by severe acute respiratory syndrome coronavirus-2 (SARS-CoV-2). J Med Virol 2020; 92: 699-702.

4. Wu Y, Xu X, Yang L, Liu C, Yang C. Nervous system damage after COVID-19 infection: presence or absence? Brain Behav Immun 2020; 87: 55.

5. Moriguchi T, Harii N, Goto J, Harada D, Sugawara H, Takamino J, et al. A first case of meningitis/encephalitis associated with SARS-Coronavirus-2. Int J Infect Dis 2020; 94: 55-58.

6. Özçelik Korkmaz M, Eğilmez OK, Özçelik MA, Güven M. Otolaryngological manifestations of hospitalised patients with confirmed COVID-19 infection. Eur Arch Otorhinolaryngol 2021; 278: 1675-1685.

7. Ralli M, Di Stadio A, Greco A, de Vincentiis M, Polimeni A. Defining the burden of olfactory dysfunction in COVID-19 patients. Eur Rev Med Pharmacol Sci 2020; 24: 3440-3441.
8. Lechien JR, Chiesa-Estomba CM, De Siati DR, Horoi M, Le Bon SD, Rodriguez A, et al. Olfactory and gustatory dysfunctions as a clinical presentation of mild-to-moderate forms of the coronavirus disease (COVID-19): a multicenter European study. Eur Arch Otorhinolaryngol 2020; 277: 2251-2261.

9. Giacomelli A, Pezzati L, Conti F, Bernacchia D, Siano M, Oreni L, et al. Self-reported olfactory and taste disorders in patients with severe acute respiratory coronavirus 2 infection: a crosssectional study. Clin Infect Dis 2020; 71: 889-890.

10. Spadera L, Viola P, Pisani D, Scarpa A, Malanga D, Sorrentino $\mathrm{G}$, et al. Sudden olfactory loss as an early marker of COVID-19: a nationwide Italian survey. Eur Arch Otorhinolaryngol 2021; 278: 247-255.

11. Chiarella G, Pizzolato R, Malanga D, Pisani D, Abenavoli L, Viola P. Prevention of COVID-19 infection in the medical population: possible help from anosmia? Rev Recent Clin Trials 2020; 15: 244-245.

12. Kennedy PG. Herpes simplex virus type 1 and Bell's palsy-a current assessment of the controversy. J Neurovirol 2010; 16: $1-5$.

13. Mateer EJ, Huang C, Shehu NY, Paessler S. Lassa fever-induced sensorineural hearing loss: a neglected public health and social burden. PLoS Negl Trop Dis 2018; 12: e0006187.

14. Kilic O, Kalcioglu MT, Cag Y, Tuysuz O, Pektas E, Caskurlu $\mathrm{H}$, et al. Could sudden sensorineural hearing loss be the sole manifestation of COVID-19? An investigation into SARS-COV-2 in the etiology of sudden sensorineural hearing loss. Int J Infect Dis 2020; 97: 208-211.

15. Karabulut B, Sürmeli M, Bozdağ Ş, Deveci İ, Doğan R, Oysu Ç. Effect of hyperbilirubinemia on medial olivocochlear system in newborns. J Int Adv Otol 2019; 15: 272-276.

16. Culhaoglu B, Erbek SS, İnce DA, Ecevit AN, Erbek S. Medial olivary complex reflex in term newborns with hyperbilirubinemia. Int J Pediatr Otorhinolaryngol 2021; 147: 110777.

17. Yılmaz Ş, Taş M, Bulut E. Contralateral suppression of transient evoked otoacoustic emissions in children with phonological disorder. Balkan Med J 2021; 38: 127-132.

18. Mattsson TS, Lind O, Follestad T, Grøndahl K, Wilson W, Nordgård S. Contralateral suppression of otoacoustic emissions in a clinical sample of children with auditory processing disorder. Int J Audiol 2019; 58: 301-310.

19. Aksoy ED, Culhaoğlu B, Öcal FCA, Erbek SS, Erbek HS. Does the efferent auditory system have a role in children with specific learning disabilities? Turk Arch Otorhinolaryngol 2019; 57: 30-33.

20. Mustafa MWM. Audiological profile of asymptomatic Covid-19 PCR-positive cases. Am J Otolaryngol 2020; 41: 102483.

21. Perret M, Bernard A, Rahmani A, Manckoundia P, Putot A. Acute labyrinthitis revealing COVID-19. Diagnostics (Basel) 2021; 11: 482.

22. Celik T, Simsek A, Koca CF, Aydin S, Yasar S. Evaluation of cochlear functions in infants exposed to SARS-CoV-2 intrauterine. Am J Otolaryngol 2021; 42: 102982. 